\title{
Formulation of Forming Load in V-Bending
}

\author{
Yuki Koumura, Zhigang Wang \\ Dept. of Mechanical Engineering, Gifu University, 1-1 Yanagido, Gifu, 501-1193, Japan
}

\begin{abstract}
A novel method is described to calculate the forming load in V-bending by a press brake. The data of forming load are collected by FEM analysis. With an increase of the punch stroke in V-bending, the forming load increases gradually after the elastic limit, and then decreases after showing the maximum value. The proposal formulation to trace the variations in the forming load curve includes the calculating method of the load of the elastic limit, the maximum load in air bending and the variations of the forming load before/after the bending stroke of the maximum load. The calculated precision is confirmed by comparing with the measured load-stroke curves in Vbending with a press brake.
\end{abstract}

\section{Introduction}

V-bending by a press brake is a common method for small lot production of sheet metal parts [1]. The most important issue in $\mathrm{V}$-bending is to decide the punch stroke to obtain the product angle precisely [2]. However, the proper punch stroke is difficult to predict accurately because it depends sensitively on mechanical properties and the thickness of the sheet metal and the width of the die [3]. Although some methods to identify mechanical properties of a sheet being bent from real time measurements have been developed, the performance is still not satisfactory for a high end machine [4-7]. The mainstream is the adjustment system with laser sensors [8], but the method is expensive because a single press uses many tools at the same time to finish a part.

In previous papers [9-11], the proper punch stroke was formulated based on geometric modeling and the numerical results of FEM analysis. The feature of the formulation is based on two new concepts; bend factor and inflection point. The bend factor is the length of bending round part of the product and depends on the mechanical properties of the sheet and the ratio of the width of die to the sheet thickness. The inflection point is the point that the bending angle under punch loading equals to the angle of the die groove. A series of experiments under various conditions showed that the control system based on the formulation is practical even for a high end machine. However, the estimation accuracy of the bending load is still to be improved.

Forming load in V-bending causes a deflection deformation of the flame in the press brake and results in a change of the product angle along the length direction. Thus estimation accuracy of the bending load is very important to make the proper crown in the machine bed to cancel the deflection deformation of the flame.
The aim of the present study is to improve the estimation accuracy of the bending load. Fig.1 illustrates the relationship between the bending load and stroke in $\mathrm{V}$-bending. The bending load increases gradually after the elastic limit $W_{p}$, and then decreases after showing the maximum value $W_{a}$. In order to trace the curve, the formulation is done as follows.

(1) Calculation method of point (1) where bending load shows the maximum

(2) Calculation method of point (2), the elastic limit

(3) Formulation of the curve (3) between the elastic limit point and the maximum load point

(4) Formulation of the curve (4) after the maximum load point

It should be noted that the formulation in the present paper does not include the inflection point that appears at the end of the bending process when the angle of product under bending load equals the die angle [10]

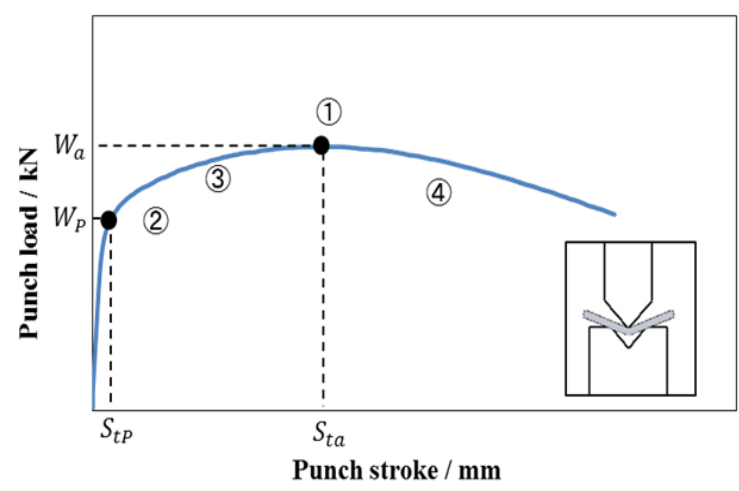

Figure 1. Relationship between punch load and punch stroke 


\section{Formulation of bending load}

\subsection{Maximum load in air bending $W_{a}$}

The equation (1) shows the formula for calculating the maximum load in air bending that is used widely in bending industries. In the present study, to improve the calculation accuracy, a correction factor $k_{m}$ that depends mainly on the sheet material is introduced and given in Table 1.

$$
\begin{gathered}
W_{a c}=\frac{k_{g} \sigma_{B} w t^{2}}{1000 \mathrm{~V}} \\
W_{a}=k_{m} W_{a c}
\end{gathered}
$$

Where $\sigma_{B}$ is tensile strength of the sheet material, $w$ is the bending length, $t$ is the sheet thickness, $V$ is the die width. As shown in the equation (3), $k_{g}$ is a factor that depends on the combination of the sheet thickness and the die width.

$$
k_{g}=-0.055 \frac{V}{t}+1.753
$$

Table 1 Value of $k_{m}$

\begin{tabular}{|c|c|c|c|}
\hline $\begin{array}{c}\text { Sheet } \\
\text { material }\end{array}$ & SPCC & A5052 & SUS304 \\
\hline$k_{m}$ & 1.17 & 0.88 & 1.09 \\
\hline
\end{tabular}

Fig.2 shows the FEM analysis results of the punch stroke $S_{t a}$ of the maximum load. $S_{t a}$ changes linearly with $V / t$. Thus $S_{t a}$ is formulated by the equation (4). As shown in Fig.3 and Fig.4, $a_{l}$ and $b_{l}$ vary simply with the sheet thickness $t$.

$$
S_{t a}=\mathrm{a}_{1}(t) \frac{V}{t}+b_{1}(t)
$$

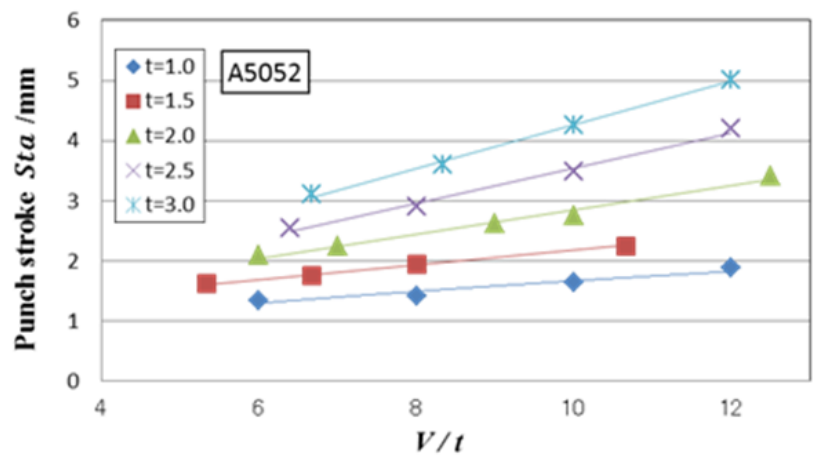

Figure 2. Relationship between punch stroke $S_{t a}$ and $V / t$

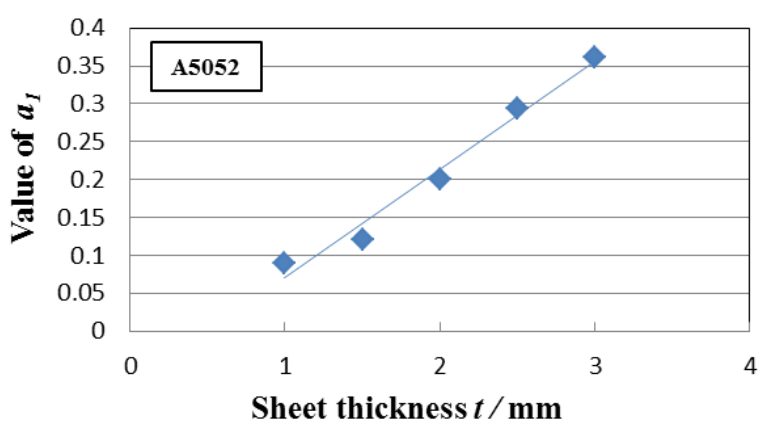

Figure 3. Relationship between value of $a_{1}$ and sheet thickness

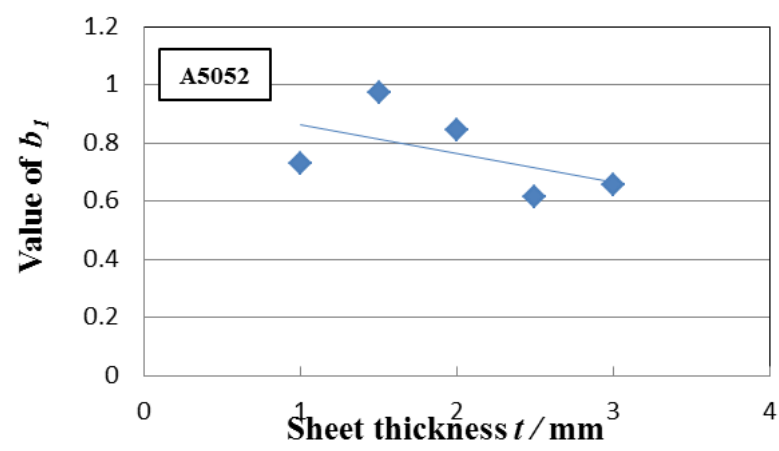

Figure 4. Relationship between value of $b_{1}$ and sheet thickness

\subsection{Elastic limit load $W_{p}$}

Fig.5 shows FEM analysis results of $W_{p}$ for different $V / t$. Based on the results, $W_{p}$ is expressed by the equation (5) As shown in Fig.6 and Fig.7, $a_{2}$ and $b_{2}$ vary simply with the sheet thickness $t$.

$$
W_{p}=a_{2}(t) \frac{V}{t}+b_{2}(t)
$$

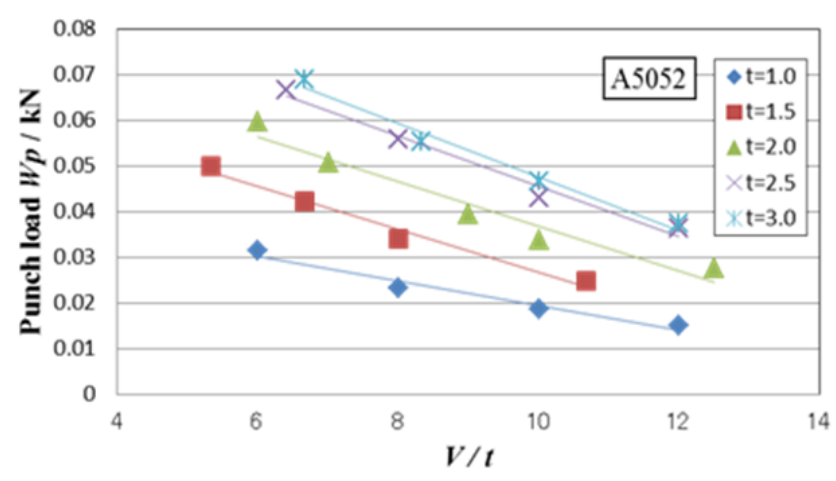

Figure 5. Relation between punch load $W_{p}$ and $V / t$ 


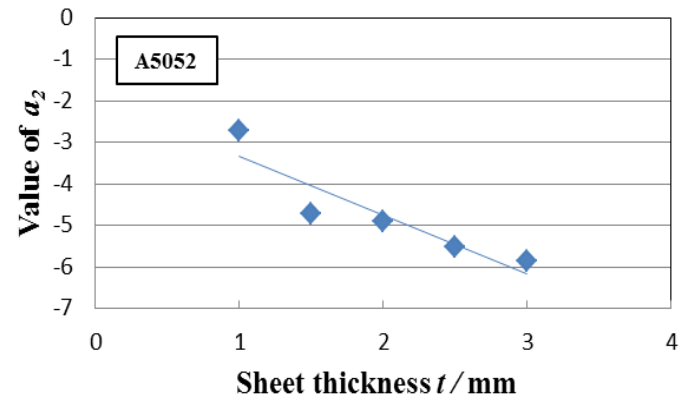

Figure 6. Relationship between value of $a_{2}$ and sheet thickness

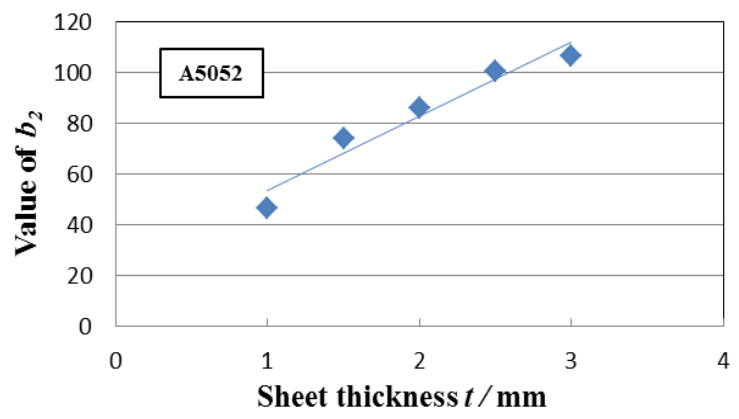

Figure 7. Relationship between value of $b_{2}$ and sheet thickness

Fig.8 shows FEM analysis results of $S_{t p}$ for different $V / t$. Based on the results, $S_{t p}$ is expressed by the equation (6). As shown in Fig.9 and Fig.10, $c_{2}$ and $d_{2}$ vary simply with the sheet thickness $t$.

$$
S_{t p}=c_{2}(t) \frac{V}{t}+d_{2}(t)
$$

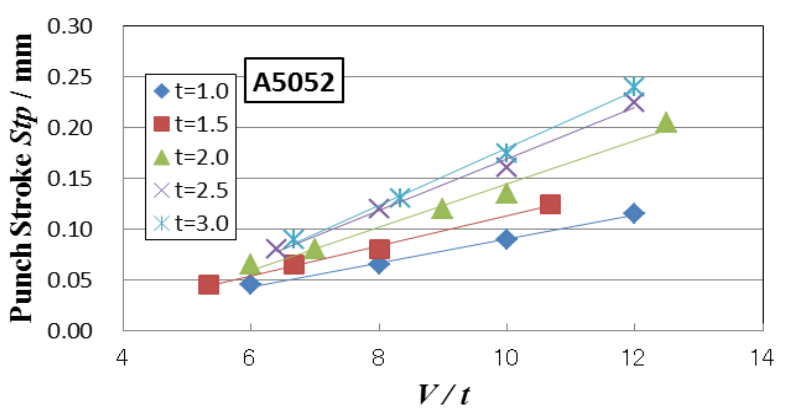

Figure 8. Relationship between punch stroke $S_{t p}$ and $V / t$

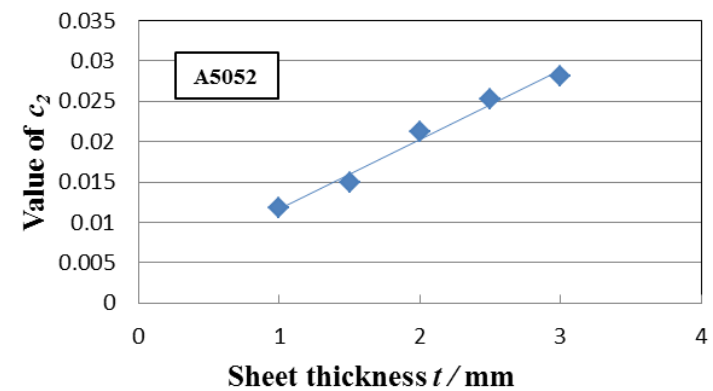

Figure 9. Relationship between value of $c_{2}$ and sheet thickness

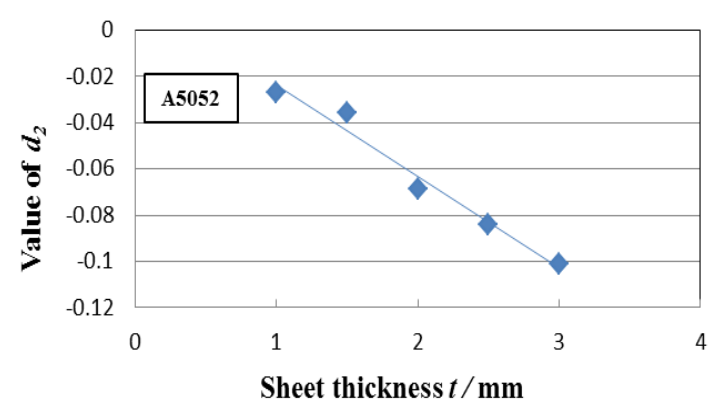

Figure 10. Relationship between value of $d_{2}$ and sheet thickness

\subsection{Bending load $W_{3}$ between the elastic limit and the maximum load point}

The formulation of relationship between the bending load $W_{3}$ and the punch stroke is difficult, the formulation of relationship between the angle of product and the punch stroke is considered. As reported in the previous paper [9], the stroke $S_{t}$ for the angle of product $\theta$ can be calculated by the equation (7).

$$
\begin{aligned}
S_{t} & =\left(t+R_{d}+\frac{A}{\pi-\left(\frac{\pi}{180} \times \theta\right)}\right)\left(1-\frac{1}{\sin \frac{\theta}{2}}\right) \\
& +\left\{\frac{V}{2}+R_{d} \tan \left(45-\frac{\emptyset}{4}\right)\right\} \cot \frac{\theta}{2}+S_{t p}
\end{aligned}
$$

Where $R_{d}$ is the radius of the die shoulder, $A$ is the bend factor, $\varnothing$ is the angle of die groove.

Fig.11 shows FEM analysis results of $W_{3} / W_{a}$ as a function of $(180-\theta) / 180$. Thus $W_{3}$ can be expressed by the equation (8). As shown in Fig.12 and Fig.13, $a_{3}$ and $b_{3}$ vary simply with $V / t$.

$$
W_{3}=a_{3}\left(\frac{V}{t}\right)\left(\frac{180-\theta}{180}\right)^{b_{3}\left(\frac{V}{t}\right)} W_{a}
$$

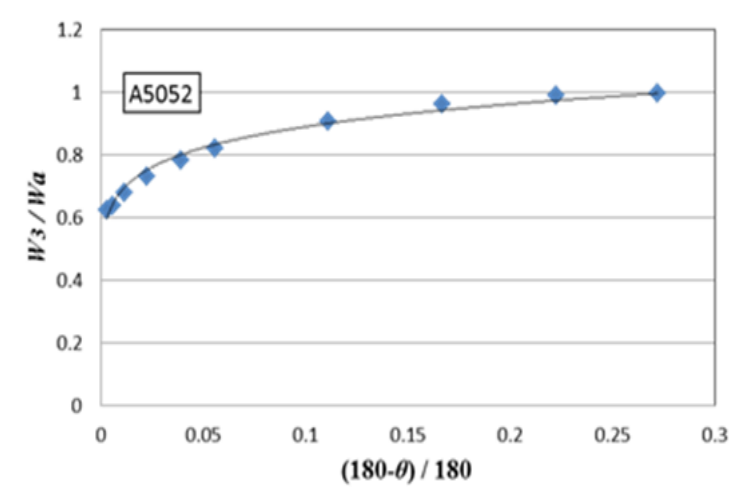

Figure 11. Relationship between $W_{3} / W_{a}$ and $(180-\theta) / 180$ 


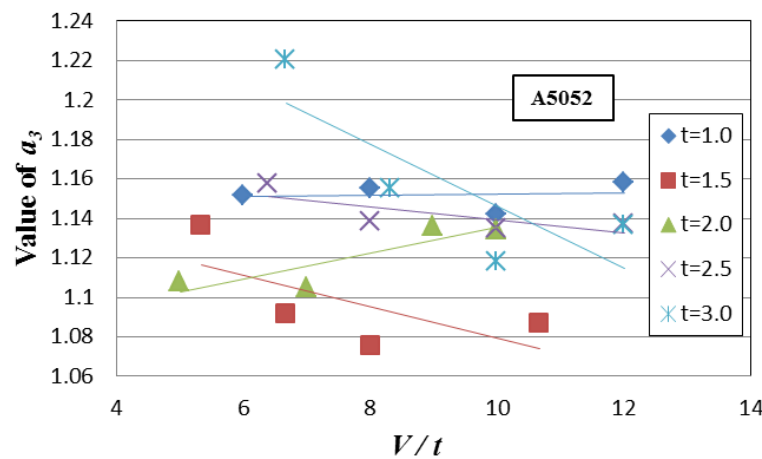

Figure 12. Relationship between value of $a_{3}$ and $V / t$

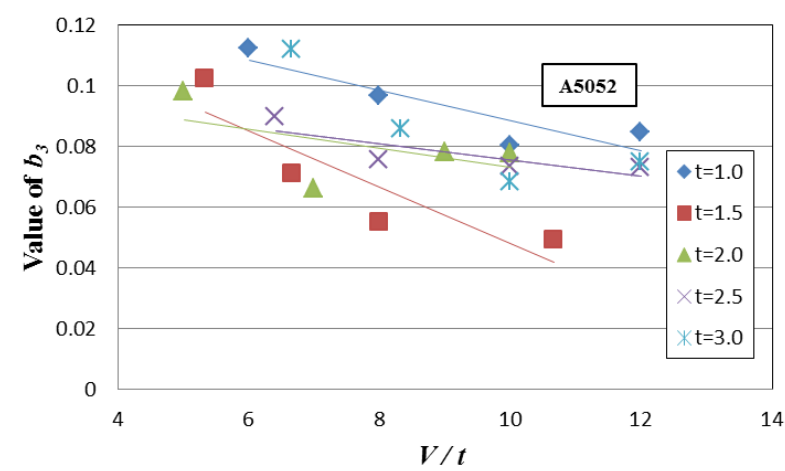

Figure 13. Relationship between value of $b_{3}$ and $V / t$

\subsection{Bending load $W_{4}$ after the maximum load point}

Fig.14 shows FEM analysis results of $W_{4} / W_{a}$. Therefore $W_{4}$ is expressed by the equation (9). As shown in Figs.15 $\mathbf{- 1 7}, a_{4}, b_{4}$ and $c_{4}$ vary simply with $V / t$.

$$
W_{4}=W_{a}\left\{a_{4}\left(\frac{V}{t}\right)\left(\frac{180-\theta}{180}\right)^{2}+b_{4}\left(\frac{V}{t}\right)\left(\frac{180-\theta}{180}\right)+c_{4}\left(\frac{V}{t}\right)\right\}
$$

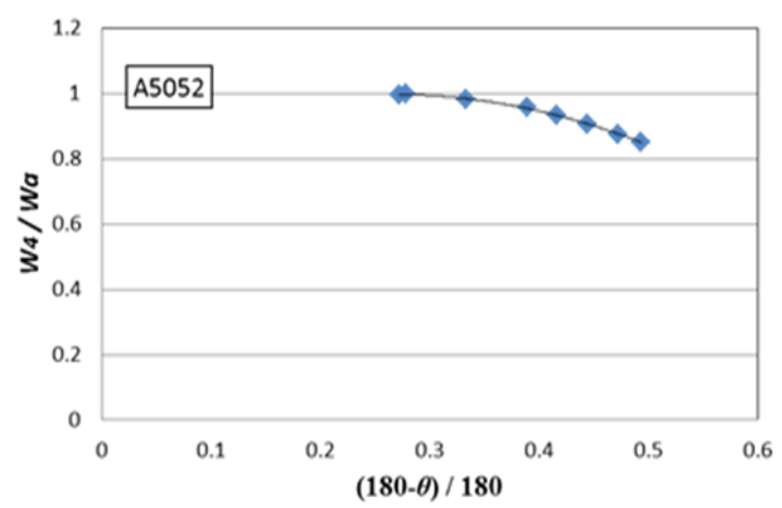

Figure 14. Relationship between $W_{4} / W_{a}$ and (180- $\left.\theta\right) / 180$

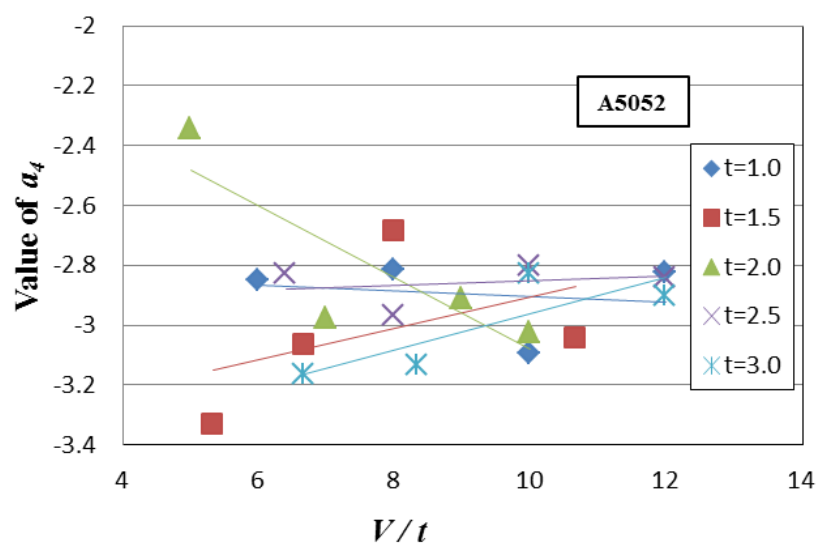

Figure 15. Relationship between value of $a_{4}$ and $V / t$

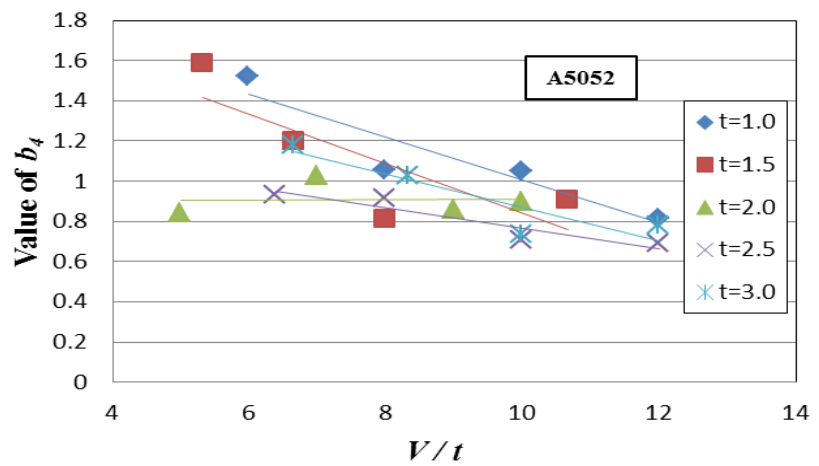

Figure 16. Relationship between value of $b_{4}$ and $V / t$

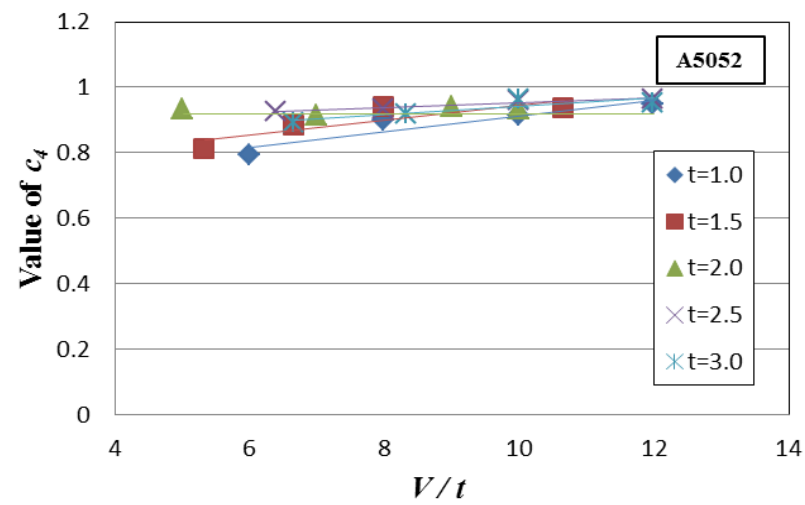

Figure 17. Relationship between value of $c_{4}$ and $V / t$

\subsection{Effect of friction on bending load}

The above mentioned data of FEM analysis is obtained by a commercial code DEFORM-2D. In the FEM simulation, friction coefficient is assumed zero to ensure the simulation stability and shorten the calculation time. The increase in the bending load due to friction is taken into account by using the equation (10).

$$
\begin{gathered}
\Delta W=\frac{\mu W}{\mu+\cot \beta} \\
\beta=90^{\circ}-\frac{\theta}{2}
\end{gathered}
$$

Where $\mu$ is the friction coefficient between the sheet and the die. 


\section{Verification experiments}

To confirm the validity of the proposed formulation, a series of experiments are carried out by using three types of dies. Table 2 shows the experimental conditions. The punch stroke is measured with a linear gauge; the punch load is measured by a load cell. Figs.18-20 shows the comparison results. The calculated curve traces precisely the experimental curve in all cases.

Table 2 Experimental conditions

\begin{tabular}{|c|c|c|c|}
\hline \multirow{3}{*}{ Material } & $\begin{array}{c}\text { Die width } \\
V[\mathrm{~mm}]\end{array}$ & $\begin{array}{c}\text { Sheet } \\
\text { thickness } \\
t[\mathrm{~mm}]\end{array}$ & $\begin{array}{c}\text { Bending } \\
\text { width } \\
w[\mathrm{~mm}]\end{array}$ \\
\hline \multirow{3}{*}{ SPCC } & 8 & 1.53 & 60.09 \\
\cline { 2 - 4 } & 10 & 1.53 & 60.62 \\
\cline { 2 - 4 } & 12 & 1.52 & 60.26 \\
\hline
\end{tabular}

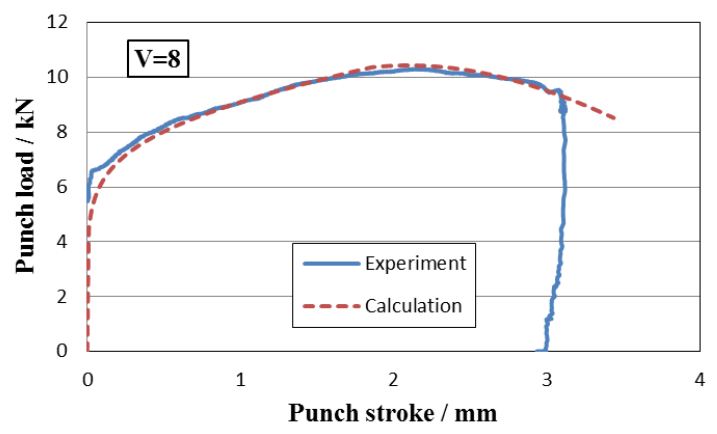

Figure 18. Comparison between measured and calculated load $(V=8)$

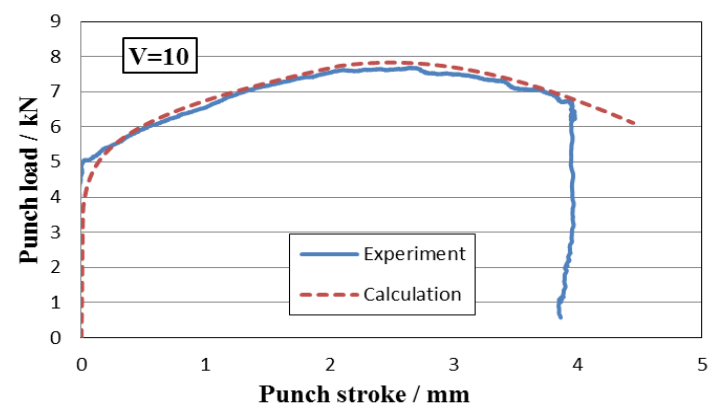

Figure 19. Comparison between measured and calculated load $(V=10)$

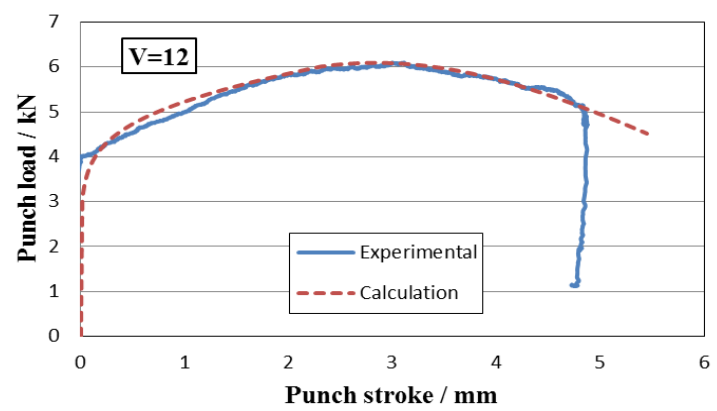

Figure 20. Comparison between measured and calculated load $(V=12)$

\section{Conclusions}

The forming load in V-bending is formulated based on the numerical results by FEM analysis. The proposal formulation includes the calculating method of the load of the elastic limit, the maximum load in air bending and the variations of the forming load before/after the bending stroke of the maximum load. The calculated precision is confirmed by comparing with the measured load-stroke curves in V-bending with a press brake.

\section{References}

1. Http://www.wila.nl/

2. Http://www.jp.trumpf.com/ja.html

3. D.C. Gossard and B.T. Allison, The proceedings of the $8^{\text {th }}$ NAMRC, 252-256 (1980).

4. K.A. Stelson and D.C. Gossard, Trans. of the ASME, Journal of Engineering for Industry, 104, 389393(1982).

5. K.A. Stelson, Trans. of the ASME, Journal of Engineering for Industry, 105, 45-53 (1983).

6. S. Kim and K.A. Stelson, Trans. of the ASME, Journal of Engineering for Industry, 110, 218-222 (1988).

7. A. Chandra, Trans. of the ASME, Journal of Engineering for Industry, 109, 265-273 (1987).

8. Http://www.amada.co.jp/

9. Z.G. Wang, S. Ikeido, and N. Kanada, The proceedings of the 2011 Japanese spring conference for the technology of plasticity, 299-300 (2011).

10. Z.G. Wang, S. Ikeido, and N. Kanada, The proceedings of the 2011 Japanese spring conference for the technology of plasticity, 301-302 (2011).

11. Z.G. Wang, S. Ikeido, and N. Kanada, The proceedings of the 2011 Japanese spring conference for the technology of plasticity, 303-304 (2011). 